\title{
The Glasgow prognostic score: Useful information when prescribing palliative radiotherapy
}

\author{
CARSTEN NIEDER $^{1,2}$, BÅRD MANNSÅKER ${ }^{2}$, ASTRID DALHAUG $^{1,2}$, \\ ADAM PAWINSKI $^{2}$ and ELLINOR HAUKLAND ${ }^{1,2}$ \\ ${ }^{1}$ Department of Clinical Medicine, Faculty of Health Sciences, UiT - The Arctic University of Norway, \\ N-9037 Troms $\varnothing ;{ }^{2}$ Department of Oncology and Palliative Medicine, Nordland Hospital, N-8092 Bod $\varnothing$, Norway
}

Received May 5, 2016; Accepted February 10, 2017

DOI: $10.3892 /$ mco.2017.1228

\begin{abstract}
The purpose of the present retrospective study was to investigate whether a score reflecting systemic inflammatory processes [the Glasgow Prognostic Score (GPS)] provides relevant information for radiation oncologists. GPS is a three-tiered score [0: normal C-reactive protein (CRP) and albumin; 1: one abnormal result; 2: increased CRP and low albumin]. Correlations between disease type and extent, resource utilization, survival and GPS were analyzed in 703 patients. In the subgroup with GPS 2, significantly higher rates of lung, adrenal gland and liver metastases were observed. An increasing GPS score was associated with a higher likelihood of anemia, leukocytosis and thrombocytosis. Comparable findings were made regarding utilization of palliative care resources, need for blood transfusion and intravenous administration of antibiotics. Compared with GPS 0 or 1 , more patients with GPS 2 did not complete their prescribed course of radiotherapy. One-third of patients with GPS 2 received treatment during the final month of life. Multivariate analysis demonstrated that GPS was a significant prognostic factor for overall survival (median, 479, 136, and 61 days, for GPS 0 , 1 and 2, respectively). In patients with GPS 2 and additional leukocytosis, the median survival was 38 days. In conclusion, GPS provides important prognostic information. This biomarker-based score may be considered for deciding fractionation, and should be validated further.
\end{abstract}

\section{Introduction}

Prognostic information is essential when choosing between treatment regimens with different levels of intensity, and when stratifying patients who participate in clinical studies. Often,

Correspondence to: Professor Carsten Nieder, Department of Oncology and Palliative Medicine, Nordland Hospital, N-8092 Bod $\varnothing$, Norway

E-mail: carsten.nieder@nlsh.no

Key words: radiation therapy, palliative radiotherapy, biomarkers, prognostic score, albumin, C-reactive protein different items, which contribute information, are combined into a clinically useful tool, e.g., a staging system or prognostic score (1). In patients with progressive cancer, the prognosis changes during the disease trajectory (2). If an assessment could be based on factors that are easy to measure and inexpensive, clinical decision-making would be improved, particularly in low-income countries with limited health care resources. In this context, the Glasgow prognostic score (GPS) might be an excellent tool (3). It is based on measurement of the levels of serum albumin and C-reactive protein (CRP), reflects inflammatory processes that may perpetuate tumor progression, and has been shown to correlate with survival in patients with lung, gastrointestinal, prostate and renal cancer (4-6). Surprisingly little research has been performed regarding its potential role in patients who receive palliative radiotherapy. As the role of palliative radiotherapy in patients with very short survival has recently come under scrutiny (7-9), in the present study, the ability of GPS to predict survival in a large contemporary cohort of patients was examined.

\section{Patients and methods}

A retrospective analysis of the records of 873 consecutive patients with metastatic or otherwise incurable cancer who received palliative radiotherapy at a single institution (Nordland Hospital, Bodø, Norway) was performed. Due to their different biological behavior, hematological and primary brain malignancies were not included. Treatment was initiated during the time period between 20 June 2007 (the date of opening of the institution's radiotherapy facility) and 31 December 2011. Medical records and treatment details were abstracted from the hospital's electronic patient record (EPR) system. The survival status and date of death, or last follow-up, of the patients were obtained from the EPR during September 2014, resulting in at least 2.5 years of follow-up for surviving patients. IBM SPSS Statistics 21.0 (IBM SPSS, Armonk, NY, USA) was used to evaluate the association between survival, GPS and other prognostic factors. Only blood test results obtained within 1 week prior to the radiotherapy were considered. For albumin, the institutional lower limit was $36 \mathrm{~g} / \mathrm{l}$. The cut-off for CRP was $8 \mathrm{mg} / \mathrm{l}$. Overall, 703 patients had available blood tests, and were eligible for the present study. The Eastern Cooperative Oncology Group 
(ECOG) performance status (PS) was registered routinely at the time of consultation in conjunction with radiotherapy (10). Actuarial survival curves from the first day of radiotherapy were generated using the Kaplan-Meier method, and compared using the log-rank test. For multivariate analysis of survival, Cox regression analysis was used (the forward stepwise method). All factors with a significant P-value in univariate log-rank tests were carried forward to multivariate regression analysis. Associations between different variables of interest were assessed with the Chi-square and Fisher exact probability tests. In two-sided tests, $\mathrm{P}<0.05$ was considered to indicate a statistically significant difference.

\section{Results}

Baseline characteristics in 703 patients. The median age was 68 years (range, 31-95 years). The median time from first cancer diagnosis to palliative radiotherapy was 2 years (range, 0-30 years). In patients with distant metastases, the median interval between diagnosis of metastatic disease and palliative radiotherapy was 7 months (range, 0-149 months). Common treatment indications included skeletal metastases (57\%), brain metastases (16\%), thoracic symptoms from lung cancer (12\%), and lymph node metastases (9\%). The most common fractionation regimen was 3 Gy x 10 (45\%), followed by 5-7 fractions of 4 Gy (22\%) and single-fraction 8 Gy x 1 (8.5 Gy x 2 for lung cancer) in $15 \%$ of the cases. Stereotactic radiotherapy was not available. The majority of the patients had prostate cancer $(26 \%)$, non-small cell lung cancer (NSCLC, 22\%) or breast cancer $(13 \%)$ as primary tumors.

GPS reflects prognostically important disease characteristics. A total of $34 \%$ of the patients had normal CRP and albumin scores (GPS 0). Of the total patients, 19\% had abnormal CRP and albumin (GPS 2). The remaining $47 \%$ of the patients had GPS 1 (in $96 \%$ of cases resulting from an elevated level of CRP; in 4\%, due to a low level of albumin). As shown in Table I, the majority of the patients with GPS 0 had a good PS (ECOG 0 or 1), whereas most patients with GPS 2 had a poor PS (ECOG 3 or 4; $\mathrm{P}=0.0001$ ). Significant differences were also observed regarding the primary tumor type. Primary tumors that were frequently associated with GPS 2 were pancreatic cancer $(40 \%$ of these patients had GPS 2), bladder cancer (30\%), colorectal cancer (28\%), and NSCLC $(26 \%)$. The pattern of metastatic disease also correlated with GPS. Patients with GPS 2 had markedly higher rates of adrenal gland, lung and liver metastases. Increasing levels of GPS were correlated with an increasing likelihood of documented disease progression outside of the radiotherapy target volumes(s) at the time of the last assessment prior to radiotherapy $(\mathrm{P}=0.0001)$. Anemia was more common in patients with a higher GPS $(\mathrm{P}=0.0001)$. Comparable correlations were detected for leukocytosis and high platelet counts $(\mathrm{P}=0.0001)$. Increasing GPS was also associated with a low level of serum creatinine, a surrogate marker of reduced muscle mass, or cachexia $(\mathrm{P}=0.0001)$.

GPS reflects resource utilization and predicts early death. As shown in Table II, GPS was associated with the utilization of palliative care resources, the number of blood transfusions and intravenous treatment with antibiotics. A larger proportion of the patients with GPS 2 were unable to complete their prescribed course of radiotherapy. One-third of patients with GPS 2 received palliative radiotherapy in the terminal phase (final month of life). Patients with GPS 2 were less often treated with long-course radiotherapy, defined as $\geq 10$ fractions $(46 \%$, compared with $62 \%$ in the subgroup with GPS 1 , and $72 \%$ of those with GPS 0; $\mathrm{P}=0.0001$ ).

Survival. The median survival rate was 183 days (6 months). As shown in Fig. 1, GPS significantly correlated with survival (median 479, 136, and 61 days, respectively, for GPS 0, 1 and 2; $\mathrm{P}=0.0001)$. Therefore GPS was entered into a multivariate Cox regression model, together with all the other prognostic factors, which achieved a $\mathrm{P}$-value $<0.05$ in univariate log-rank tests [age as a continuous variable, ECOG PS, primary tumor type, anemia, leukocytosis, low serum creatinine, progressive disease outside of target volume(s), brain metastases, liver metastases, lung metastases, bone metastases, and adrenal gland metastases]. Seven out of these 13 variables were revealed to be independent prognostic factors for survival: GPS, ECOG PS, brain metastases, liver metastases, bone metastases, progressive disease outside of target volume(s), and leukocytosis (all with $\mathrm{P} \leq 0.001$ ).

On the one hand, the median survival of patients with GPS 2 was short, and administration of radiotherapy during the last month of life was not unusual. On the other hand, survival in this subgroup was too heterogeneous to conclude that all these patients were poor candidates for radiotherapy. An appreciable number of them survived long enough to benefit from and experience symptom improvement. Thus, additional variables are required in order to define a subgroup with uniformly short survival. As mentioned above, these variables should be easy to assess and inexpensive in order to decide upon which model would be applicable to practice settings in countries with limited resources. Factoring in ECOG PS was clearly a way forward. Figs. 2 and 3 show the usefulness of combining GPS and ECOG PS, at least in patients with GPS 0 and 1. However, in the relevant target group of patients with GPS 2, the survival data did not reveal significant differences (Fig. 4). Table III shows the median survival of patients with different ECOG PS stratified by GPS. The two groups with the shortest survival rates were patients with ECOG PS 3 and GPS 2, and patients with ECOG PS 4, irrespective of GPS. The second variable not requiring any additional resources (such as, e.g., imaging) was leukocytosis. This may be evaluated from the drawing of the same blood required to assign GPS. As shown in Fig. 5, patients with GPS 2 and leukocytosis had a median survival of 38 days, as compared with 89 days in those with a normal leukocyte count $(\mathrm{P}=0.007)$. Only $20 \%$ of the patients with three abnormal biomarkers were alive after 3 months. Comparable associations were observed in patients with GPS 1 (median, 94 vs. 192 days) and GPS 0 (median, 162 vs. 635 days).

Models with a different CRP cut-off. GPS does not take into account the magnitude of CRP increase: Assignment is based solely on normal vs. increased CRP. Therefore, the analyses in the present study were expanded, and different levels of an increase in CRP (30, 60 or $90 \mathrm{mg} / \mathrm{l})$ were examined. However, survival prediction did not improve in these analyses requiring certain levels of an increase in CRP (results not shown). Albumin levels were less variable. Almost all patients with low levels of albumin had measurements of 30-35 g/l. Therefore, stratified analyses could not be meaningfully performed. 
Table I. Baseline characteristics stratified by GPS scores of the patients $(n=703)$.

\begin{tabular}{|c|c|c|c|c|c|c|c|}
\hline Parameter & 0 & $\%$ & 1 & $\%$ & 2 & $\%$ & P-value \\
\hline \multicolumn{8}{|l|}{ Gender } \\
\hline Female & 90 & 37 & 114 & 35 & 48 & 37 & \\
\hline Male & 151 & 63 & 216 & 65 & 83 & 63 & 0.77 \\
\hline \multicolumn{8}{|l|}{ Age, years } \\
\hline$<60$ & 47 & 20 & 71 & 21 & 24 & 18 & 0.7 \\
\hline$\geq 80$ & 38 & 16 & 44 & 13 & 20 & 15 & 0.46 \\
\hline \multicolumn{8}{|l|}{ ECOG PS } \\
\hline 0 & 41 & 17 & 35 & 11 & 2 & 2 & \\
\hline 1 & 111 & 46 & 98 & 30 & 10 & 8 & \\
\hline 2 & 63 & 26 & 124 & 37 & 46 & 35 & \\
\hline 3 & 25 & 10 & 61 & 18 & 63 & 48 & \\
\hline 4 & 0 & 0 & 13 & 4 & 10 & 8 & 0.0001 \\
\hline \multicolumn{8}{|l|}{ Type of cancer } \\
\hline Prostate cancer & 91 & 38 & 71 & 21 & 21 & 16 & \\
\hline Breast cancer & 43 & 18 & 31 & 9 & 15 & 11 & \\
\hline Non-small cell lung cancer & 34 & 14 & 82 & 25 & 41 & 31 & \\
\hline Small cell lung cancer & 15 & 6 & 24 & 7 & 4 & 3 & \\
\hline Colorectal cancer & 10 & 4 & 23 & 7 & 13 & 10 & \\
\hline Pancreatic cancer & 1 & 0 & 5 & 2 & 4 & 3 & \\
\hline Bladder cancer & 7 & 3 & 24 & 7 & 13 & 10 & \\
\hline Malignant melanoma & 9 & 4 & 11 & 3 & 2 & 2 & \\
\hline Kidney cancer & 11 & 5 & 35 & 11 & 8 & 6 & \\
\hline Other primary tumor & 20 & 8 & 25 & 8 & 10 & 8 & 0.0001 \\
\hline \multicolumn{8}{|l|}{ Metastases } \\
\hline Bone $^{a}$ & 162 & 68 & 222 & 67 & 102 & 78 & 0.06 \\
\hline Brain $^{a}$ & 50 & 21 & 67 & 20 & 22 & 17 & 0.61 \\
\hline Liver $^{\mathrm{a}}$ & 40 & 17 & 77 & 23 & 37 & 28 & 0.028 \\
\hline Lung $^{\mathrm{a}}$ & 41 & 17 & 97 & 29 & 46 & 35 & 0.0001 \\
\hline Adrenal $^{\mathrm{a}}$ & 14 & 6 & 38 & 11 & 25 & 19 & 0.0001 \\
\hline No distant & 28 & 4 & 34 & 5 & 8 & 1 & 0.23 \\
\hline Progressive disease outside of RT field & 97 & 42 & 189 & 58 & 90 & 73 & 0.0001 \\
\hline Anemia $^{\mathrm{b}}$ & 101 & 42 & 223 & 68 & 116 & 89 & 0.0001 \\
\hline Leukocytosis $^{c}$ & 31 & 13 & 83 & 25 & 56 & 44 & 0.0001 \\
\hline Thrombocytosis $^{\mathrm{d}}$ & 34 & 14 & 105 & 33 & 50 & 40 & 0.0001 \\
\hline \multicolumn{8}{|l|}{ Serum creatinine } \\
\hline Low $^{\mathrm{e}}$ & 16 & 2 & 49 & 7 & 36 & 5 & $0.0001^{\mathrm{e}}$ \\
\hline High $^{\mathrm{e}}$ & 27 & 4 & 58 & 8 & 19 & 3 & \\
\hline No previous systemic therapy & 88 & 39 & 131 & 42 & 58 & 48 & 0.26 \\
\hline
\end{tabular}

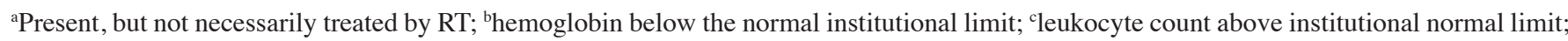

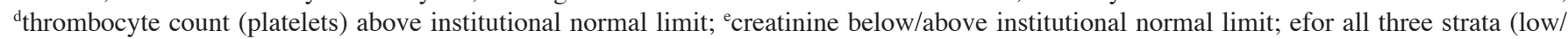
normal/high). ECOG, Eastern Cooperative Oncology Group; PS, performance status; GPS, Glasgow Prognostic Score; RT, radiotherapy.

\section{Discussion}

Irrespective of the health care setting, increasing expenses and limited budgets create serious challenges for the oncology community. Initiatives such as the 'Choosing Wisely' campaign (11) are trying to provide support to stakeholders, who are required to discriminate between cost-effective interventions and imprudent resource utilization. Although palliative radiotherapy, in general, is considered excellent value for money, the large number of available fractionation regimens and techniques, and the broad spectrum of survival outcomes, make it difficult to always ensure that the correct treatment is assigned to the right patient (12). Staging systems and prognostic scores play an invaluable role when assessing 


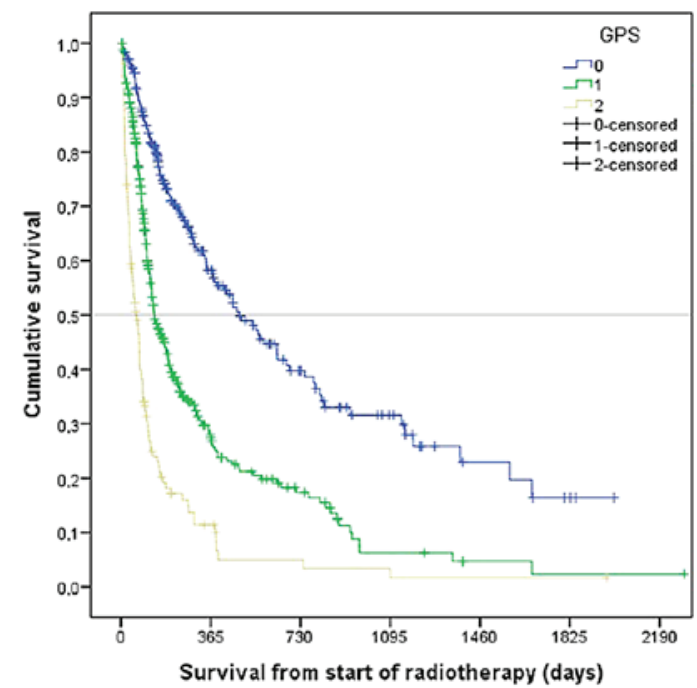

Figure 1. Actuarial overall survival after palliative radiotherapy stratified by GPS $(\mathrm{P}=0.0001, \log$-rank test). The number of patients was 241,331 , and 131 for GPS 0, 1 and 2, respectively. GPS, Glasgow prognostic score.

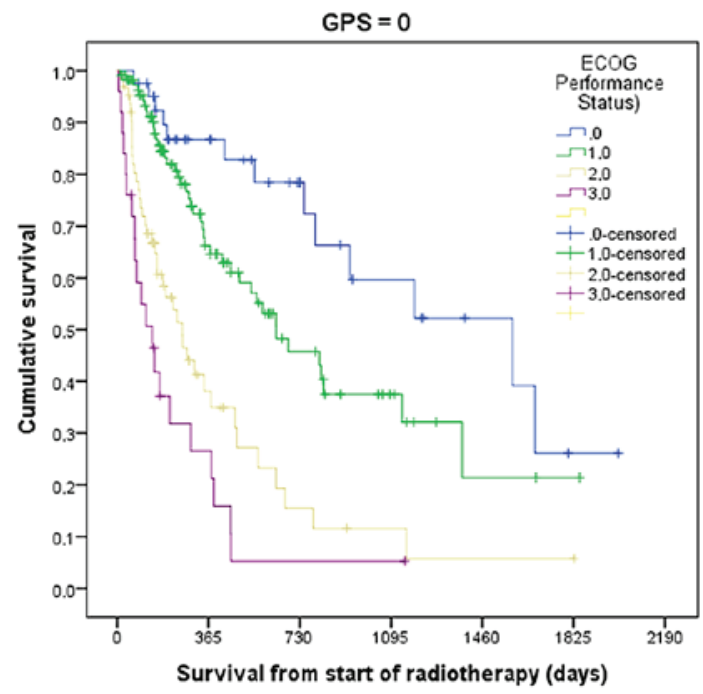

Figure 2. Actuarial overall survival following palliative radiotherapy in patients with GPS 0 stratified by ECOG performance status $(\mathrm{P}=0.0001$, log-rank test). The number of patients was 41, 111, 64, and 25 for ECOS performance statuses of $0,1,2$ and 3 , respectively. GPS, Glasgow prognostic score; ECOG, Eastern Cooperative Oncology Group.

individual patients and making treatment recommendations, as demonstrated by the broad adoption of brain metastases scores $(13,14)$. Particularly in patients with a limited survival expectation, decision making should not require comprehensive and expensive restaging, which could also delay the initiation of meaningful palliative interventions. These considerations are even more relevant for low-income countries. Widely available routine blood tests may provide excellent prognostic information, and value for money (15), since results are available within a few hours.

During the last decade, an inflammation-based prognostic score, GPS, has been studied in different oncology settings, e.g., in chemotherapy for NSCLC and prostate cancer $(3,6)$. This research suggests that malnourished cancer patients had a statistically significant higher CRP, neutrophil-to-lymphocyte ratio

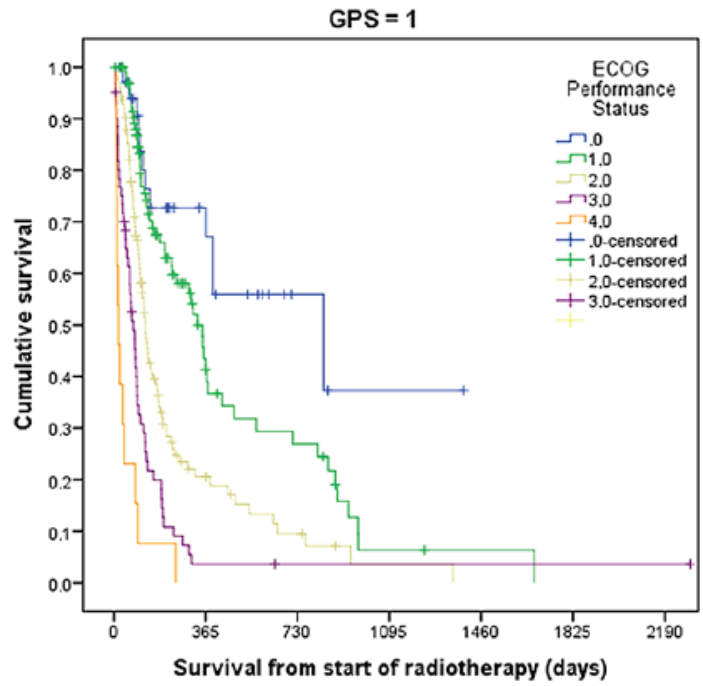

Figure 3. Actuarial overall survival following palliative radiotherapy in patients with GPS 1 stratified by ECOG performance status $(\mathrm{P}=0.001, \log$ rank test). The number of patients was 35, 98, 124, 61, and 13 for ECOS performance statuses of $0,1,2,3$, and 4, respectively. GPS, Glasgow prognostic score; ECOG, Eastern Cooperative Oncology Group.

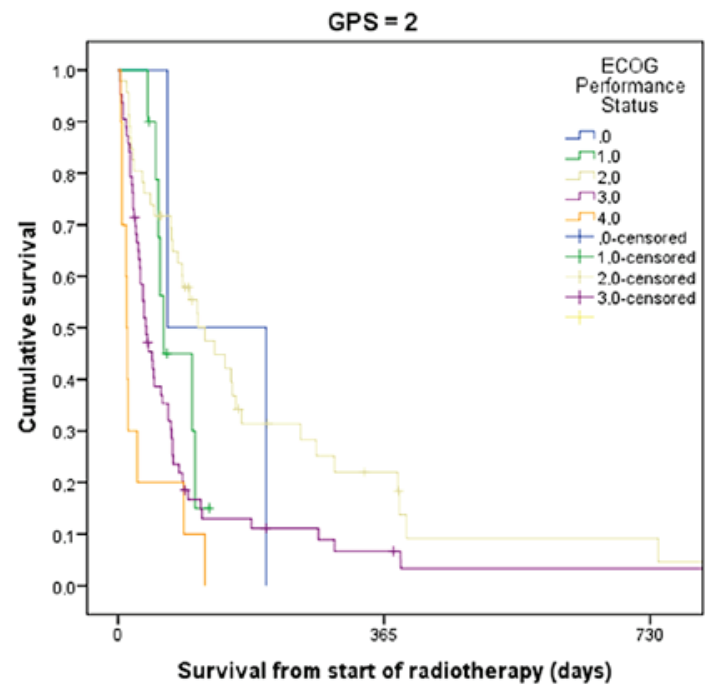

Figure 4. Actuarial overall survival following palliative radiotherapy in patients with GPS 2 stratified by ECOG performance status $(\mathrm{P}=0.3, \log$-rank test). The number of patients was 2, 10,46, 63, and 10, for ECOS performance statuses of $0,1,2,3$, and 4 , respectively. GPS, Glasgow prognostic score; ECOG, Eastern Cooperative Oncology Group.

and GPS prior to starting the chemotherapy (16). Eventually, it was shown that GPS predicts cancer survival independently of the tumor site (4). Limited data are available regarding GPS and radiotherapy (17). The latter study focused on the end-oflife care, and identified that GPS 2 was strongly associated with the receipt of radiotherapy during the last 30 days of life. The present study, to the best of the authors' knowledge, is the first comprehensive analysis of GPS in a large cohort of patients who received palliative radiotherapy. GPS was assigned retrospectively, and not used during the time period of actual patient treatments. Only a minority of patients (34\%) had normal CRP and albumin, i.e., GPS 0. Strong associations between GPS and ECOG PS were identified, comparable with earlier 
Table II. Resource utilization and treatment completion stratified by GPS scores of the patients $(n=703)$.

\begin{tabular}{lrrrrrr}
\hline Endpoint & 0 & $\%$ & 1 & $\%$ & 2 & $\%$ \\
\hline Received blood transfusion during RT & 2 & 1 & 17 & 5 & 15 & 12 \\
Received iv antibiotics during RT & 7 & 3 & 34 & 11 & 28 & 23 \\
Care by palliative team during RT & 38 & 17 & 75 & 24 & 42 & 37 \\
Pain management with continuous & 2 & 1 & 14 & 4 & 17 & 13 \\
infusion pump & & & & 0.0001 \\
Incomplete RT & 4 & 2 & 21 & 6 & 17 & 13 \\
RT during last month of life & 8 & 3 & 33 & 10 & 43 & 33 \\
\hline
\end{tabular}

RT, radiotherapy; iv, intravenous.

Table III. Median survival (days, 95\% confidence interval) in patients with different PSs stratified by GPS scores (n=702).

\begin{tabular}{lcccc}
\hline ECOS PS & No. of patients & 0 & 1 & 2 \\
\hline 0 & 78 & $1,580(686-2,474)$ & $833(58-1,608)$ & 68 (n/a; only 2 patients) \\
1 & 219 & $636(372-900)$ & $332(275-389)$ & $63(49-77)$ \\
2 & 233 & $261(163-359)$ & $126(107-145)$ & $120(70-170)$ \\
3 & 149 & $140(57-223)$ & $78(57-99)$ & $39(26-52)$ \\
4 & 23 & No patients & $19(7-31)$ & $12(9-15)$ \\
\hline
\end{tabular}

ECOG, Eastern Cooperative Oncology Group; PS, performance score; n/a, not applicable.

reports (18). It is tempting to speculate that GPS is merely a surrogate marker of tumor extent and tumor-host interactions, since GPS correlated with progression, the pattern of metastatic disease, anemia, leukocytosis and thrombocytosis. However, in multivariate analysis of prognostic factors for overall survival that included all these variables, the added value of GPS was confirmed. This finding is in line with non-radiotherapy studies $(5,19,20)$. Due to its robust prognostic value, low cost and wide availability, additional studies of GPS in different radiotherapy settings are recommended.

Previous studies also found significant associations between GPS and the chemotherapy dose adjustment, the requirement for granulocyte colony-stimulating factor support, termination of treatment due to side-effects, and fatal toxicity (21). In the present study, GPS was associated with more frequent utilization of palliative care resources, the number of blood transfusions and intravenous treatment with antibiotics. Compared with patients with GPS 0 or 1 , more patients with GPS 2 were not able to complete their prescribed course of radiotherapy. A total of $33 \%$ of the patients with GPS 2 received treatment during the final month of their life. It is therefore important to consider short-course (e.g., 8.5 Gy x 2, 4 Gy x 5) and single-fraction (8 Gy) radiotherapy regimens in patients with GPS 2. The development of prediction models specifically for the purpose of avoidance of too aggressive end-of-life care is in its infancy, and, for the time being, is an unmet requirement (22-24). A previous study suggested that performance status and GPS, used together, act synergistically, thus improving the prognostic accuracy (19). Even if this was not entirely confirmed in the present study, patients with

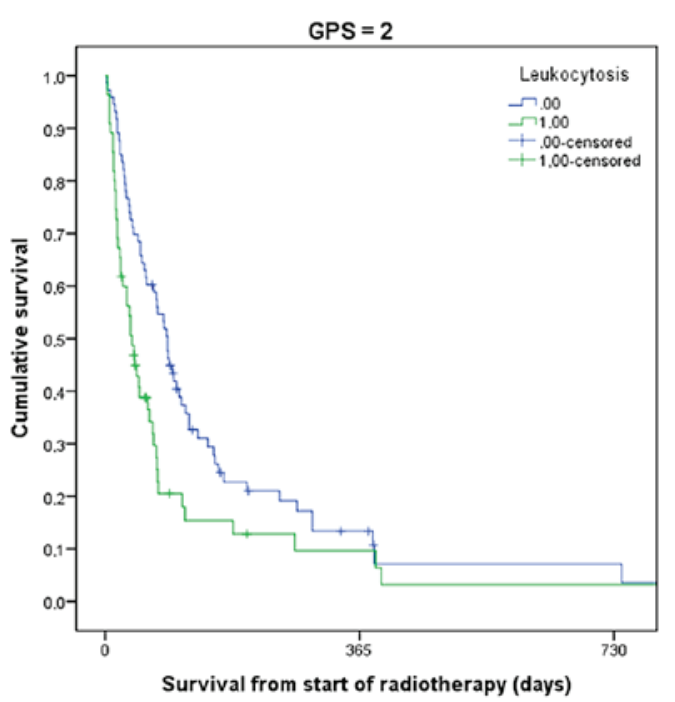

Figure 5. Actuarial overall survival following palliative radiotherapy in patients with GPS 2 stratified by leukocyte count [normal ( $n=73$ patients) vs. increased $(\mathrm{n}=55$ patients), $\mathrm{P}=0.007$, log-rank test). GPS, Glasgow prognostic score.

GPS 2 and ECOG PS 3 or 4 had short median survival rates. Possibly, the discrepancy between the two studies was caused by the small group sizes.

In the present study, it was established that it was possible to improve the prediction of short survival by combining leukocytosis and GPS 2. Patients with these two characteristics had a median survival of $>6$ weeks. This combination of variables has not been evaluated previously in the same setting. 
Considering cancer-specific survival, Proctor et al (25) demonstrated that the prognostic value of the GPS improved by the addition of neutrophil and platelet counts and a high-sensitivity CRP measurement ( $>3 \mathrm{mg} / \mathrm{l})$. Neither neutrophil counts nor high-sensitivity CRP assays were part of our standard work-up during the time period of the present study. In addition, the platelet count was not significantly associated with survival.

In conclusion, the present study has revealed that three widely available, inexpensive blood tests (CRP, albumin, and leukocyte count) provide clinically relevant prognostic information for radiation oncologists who decide upon fractionation. Further studies of the original GPS, and modified variants, are warranted.

\section{References}

1. Tseng YD, Krishnan MS, Sullivan AJ, Jones JA, Chow E and Balboni TA: How radiation oncologists evaluate and incorporate life expectancy estimates into the treatment of palliative cancer patients: A survey-based study. Int J Radiat Oncol Biol Phys 87: 471-478, 2013

2. Likhacheva A, Pinnix CC, Parikh NR, Allen PK, McAleer MF, Chiu MS, Sulman EP, Mahajan A, Guha-Thakurta N, Prabhu SS, et al: Predictors of survival in contemporary practice after initial radiosurgery for brain metastases. Int J Radiat Oncol Biol Phys 85: 656-661, 2013.

3. Forrest LM, McMillan DC, McArdle CS, Angerson WJ and Dunlop DJ: Comparison of an inflammation-based prognostic score (GPS) with performance status (ECOG) in patients receiving platinum-based chemotherapy for inoperable nonsmall-cell lung cancer. Br J Cancer 90: 1704-1706, 2004.

4. Proctor MJ, Morrison DS, Talwar D, Balmer SM, O'Reilly DS, Foulis AK, Horgan PG and McMillan DC: An inflammationbased prognostic score (mGPS) predicts cancer survival independent of tumour site: A Glasgow Inflammation Outcome Study. Br J Cancer 104: 726-734, 2011.

5. Proctor MJ, Morrison DS, Talwar D, Balmer SM, Fletcher CD, O'Reilly DS, Foulis AK, Horgan PG and McMillan DC: A comparison of inflammation-based prognostic scores in patients with cancer. A Glasgow Inflammation Outcome Study. Eur J Cancer 47: 2633-2641, 2011.

6. Linton A, Pond G, Clarke S, Vardy J, Galsky M and Sonpavde G: Glasgow prognostic score as a prognostic factor in metastatic castration-resistant prostate cancer treated with docetaxel-based chemotherapy. Clin Genitourin Cancer 11: 423-430, 2013

7. Guadagnolo BA, Liao KP, Elting L, Giordano S, Buchholz TA and Shih YC: Use of radiation therapy in the last 30 days of life among a large population-based cohort of elderly patients in the United States. J Clin Oncol 31: 80-87, 2013.

8. Murphy JD, Nelson LM, Chang DT, Mell LK and Le QT: Patterns of care in palliative radiotherapy: A population-based study. J Oncol Pract 9: e220-e227, 2013.

9. Kapadia NS, Mamet R, Zornosa C, Niland JC, D'Amico TA and Hayman JA: Radiation therapy at the end of life in patients with incurable nonsmall cell lung cancer. Cancer 118: 4339-4345, 2012.

10. Kelly CM and Shahrokni A: Moving beyond Karnofsky and ECOG performance status assessments with new technologies. J Oncol 2016: 6186543, 2016.

11. Hahn C, Kavanagh B, Bhatnagar A, Jacobson G, Lutz S, Patton C, Potters L and Steinberg M: Choosing wisely: The American Society for Radiation Oncology's top 5 list. Pract Radiat Oncol 4: 349-355, 2014
12. Olson RA, Tiwana MS, Barnes M, Kiraly A, Beecham K, Miller S, Hoegler D and Olivotto I: Use of single- versus multiple-fraction palliative radiation therapy for bone metastases: Population-based analysis of 16,898 courses in a Canadian province. Int J Radiat Oncol Biol Phys 89: 1092-1099, 2014.

13. Sperduto PW, Chao ST, Sneed PK, Luo X, Suh J, Roberge D, Bhatt A, Jensen AW, Brown PD, Shih H, et al: Diagnosis-specific prognostic factors, indexes, and treatment outcomes for patients with newly diagnosed brain metastases: A multi-institutional analysis of 4,259 patients. Int J Radiat Oncol Biol Phys 77: 655-661, 2010.

14. Gaspar L, Scott C, Rotman M, Asbell S, Phillips T, Wasserman T, McKenna WG and Byhardt R: Recursive partitioning analysis (RPA) of prognostic factors in three Radiation Therapy Oncology Group (RTOG) brain metastases trials. Int J Radiat Oncol Biol Phys 37: 745-751, 1997.

15. Partl R, Richtig E, Avian A, Berghold A and Kapp KS: Karnofsky performance status and lactate dehydrogenase predict the benefit of palliative whole-brain irradiation in patients with advanced intra- and extracranial metastases from malignant melanoma. Int J Radiat Oncol Biol Phys 85: 662-666, 2013.

16. Tan CS, Read JA, Phan VH, Beale PJ, Peat JK and Clarke SJ: The relationship between nutritional status, inflammatory markers and survival in patients with advanced cancer: A prospective cohort study. Support Care Cancer 23: 385-391, 2015.

17. Anshushaug M, Gynnild MA, Kaasa S, et al: Characterization of patients receiving palliative chemo- and radiotherapy during end of life at a regional cancer center in Norway. Acta Oncol 27: $1-8,2014$

18. Leung EY, Scott HR and McMillan DC: Clinical utility of the pretreatment glasgow prognostic score in patients with advanced inoperable non-small cell lung cancer. J Thorac Oncol 7: 655-662, 2012.

19. Laird BJ, Kaasa S, McMillan DC, Fallon MT, Hjermstad MJ, Fayers P and Klepstad P: Prognostic factors in patients with advanced cancer: A comparison of clinicopathological factors and the development of an inflammation-based prognostic system. Clin Cancer Res 19: 5456-5464, 2013.

20. Furukawa K, Shiba H, Haruki K, Fujiwara Y, Iida T, Mitsuyama Y, Ogawa M, Ishida Y, Misawa T and Yanaga K: The Glasgow prognostic score is valuable for colorectal cancer with both synchronous and metachronous unresectable liver metastases. Oncol Lett 4: 324-328, 2012

21. Gioulbasanis I, Pallis A, Vlachostergios PJ, Xyrafas A, Giannousi Z, Perdikouri IE, Makridou M, Kakalou D and Georgoulias V: The Glasgow Prognostic Score (GPS) predicts toxicity and efficacy in platinum-based treated patients with metastatic lung cancer. Lung Cancer 77: 383-388, 2012.

22. Angelo K, Norum J, Dalhaug A, Pawinski A, Aandahl G, Haukland E, Engljähringer K and Nieder C: Development and validation of a model predicting short survival (death within 30 days) after palliative radiotherapy. Anticancer Res 34: 877-885, 2014.

23. Gripp S, Mjartan S, Boelke E and Willers R: Palliative radiotherapy tailored to life expectancy in end-stage cancer patients: Reality or myth? Cancer 116: 3251-3256, 2010.

24. Nieder C, Angelo K, Dalhaug A, Pawinski A, Haukland E and Norum J: Palliative radiotherapy during the last month of life: Predictability for referring physicians and radiation oncologists. Oncol Lett 10: 3043-3049,2015.

25. Proctor MJ, Horgan PG, Talwar D, Fletcher CD, Morrison DS and McMillan DC: Optimization of the systemic inflammation-based Glasgow prognostic score: A Glasgow Inflammation Outcome Study. Cancer 119: 2325-2332, 2013. 